\title{
All-optical phase and amplitude regenerator for next- generation telecommunications systems
}

\author{
Radan Slavík $^{1^{*+}}$, Francesca Parmigiani ${ }^{1^{*}}$, Joseph Kakande ${ }^{1^{*}}$, Carl Lundström² $^{2}$, Martin Sjödin $^{2}$, \\ Peter A. Andrekson ${ }^{2}$, Ruwan Weerasuriya ${ }^{3}$, Stylianos Sygletos ${ }^{3}$, Andrew D. Ellis ${ }^{3}$, Lars Grüner-Nielsen ${ }^{4}$, \\ Dan Jakobsen ${ }^{4}$, Søren. Herstrøm ${ }^{4}$, Richard Phelan ${ }^{5}$, James O'Gorman ${ }^{5}$, Adonis Bogris ${ }^{6}$, Dimitris Syvridis ${ }^{6}$, \\ Sonali Dasgupta ${ }^{1}$, Periklis Petropoulos ${ }^{1}$, and David J. Richardson ${ }^{1}$ \\ 1. Optoelectronics Research Centre, University of Southampton, Southampton, SO17 1BJ, UK \\ 2. Photonics Laboratory, Department of Microtechnology and Nanoscience, Chalmers University of Technology, SE-412 96 Göteborg, Sweden \\ 3. Tyndall National Institute and Department of Physics, University College Cork, Cork, Ireland \\ 4. OFS, Priorparken 680, 2605 Brøndby, Denmark \\ 5. Eblana Photonics Inc., Dublin, Ireland \\ 6. University of Athens, Panepistimiopolis, Illisia, Athens, GR-15784, Greece \\ +E-mail:rxs@orc.soton.ac.uk \\ * These authors contributed equally to this work
}

Fibre optic communication systems have traditionally carried data using binary (on-off) encoding of the light amplitude. However, next generation systems will exploit both the amplitude and phase of the optical carrier to achieve higher spectral efficiencies and thus higher overall data capacities ${ }^{1,2}$. Although this approach requires highly complex transmitters and receivers, the increased capacity and many further practical benefits that accrue from a full knowledge of the amplitude and phase of the optical-field ${ }^{3}$, more than outweigh this additional hardware complexity and can greatly simplify optical network design. However, use of the complex optical-field gives rise to a new dominant limitation to system performance, namely nonlinear phase noise ${ }^{4,5}$. A device for removal of this noise therefore becomes of great technical importance. Here we report the development of the first practical ('black-box') all-optical regenerator capable of removing both phase and amplitude noise from binary phase-encoded optical communication signals.

Nonlinear phase noise arises from nonlinear interactions mainly due to Kerr nonlinearity ${ }^{4,5}$. These interactions depend on the amplitude and phase noise evolution of all signals transmitted within the fibre and may inflict serious impairments in modern telecommunication systems, where many individual wavelength channels are transmitted simultaneously. Each channel is also degraded by other sources of amplitude/phase noise generated within the transmission line including amplified spontaneous emission from optical amplifiers, quantum noise, and noise originating from other components. An ideal regenerator would suppress noise in both the signal's amplitude and phase and this would bring a two-fold impact: (i) it would regenerate the noisy signal allowing for further transmission or onward signal processing and, (ii) if installed within the transmission link, it would help to suppress the seed noise that causes nonlinear signal phase noise to build up in the first place ${ }^{4,5}$.

Regenerators of optical signals are based on either electronic or all-optical signal processing and both these approaches have their advocates. Electronic-based regeneration requires optical-toelectrical-to-optical data conversion. High data rate signals can be regenerated by exploiting well established electronic-based parallel data processing techniques. All-optical regeneration allows far more straightforward serial (rather than parallel) signal processing without the need of optical-to-electrical-to-optical data conversion. However, practically implementing this using existing technology is extremely challenging. It is worth mentioning that besides optical communications, the successful development of an all-optical version of an amplitude/phase 
regenerator would be beneficial within many other fields. As an example, all-optical logic operations on phase-encoded signals would require this functionality ${ }^{6}$; phase regeneration can be viewed as a form of analog-to-digital conversion, suggesting potential applications also. Coupled with the fact that phase-sensitive amplifiers that forms the core of our regenerator can have subquantum limit noise figures ${ }^{7}$, the device discussed here may find applications in quantum communications ${ }^{8}$, free space ultra-long haul communication (e.g. inter-satellite communications) and in metrology 9 .

To date most work on all-optical regeneration has focused on amplitude regeneration ${ }^{10,11}$. A plausible approach towards the regeneration of phase-encoded signals is thus to adopt a phase-toamplitude format conversion and then to apply amplitude regeneration ${ }^{12}$ before converting back to a phase-encoded signal. Besides being rather complex, the initial format conversion may further degrade the already-noisy signal. In addition, because such schemes operate on the intensity rather than directly on the electric field of the demodulated signal they have significantly lower tolerance to phase-noise. As a consequence direct removal of phase noise is far more desirable.

Phase regeneration can be implemented by exploiting the phase-squeezing capability of phase-sensitive amplifiers (PSA) ${ }^{13,14}$. Amplitude regeneration can be simultaneously performed by operating the amplifier in the saturated regime ${ }^{15}$. The principle of operation is shown in Fig. 1. In a phase-insensitive amplifier, such as an erbium-doped fibre amplifier (Fig. 1a), the inphase and quadrature signal components experience identical gain, and as a result the field amplitude is amplified with the phase unaffected. However, in PSAs, the two quadrature components are amplified differently (Fig. 1b). For example, in a PSA based on degenerate four wave mixing $(\mathrm{FWM})^{15}$, the in-phase component of the electric field experiences a gain $g$, while the quadrature component experiences a de-amplification by $1 / g$ (Fig. 1b). Subsequently, the output phase of the amplified signal is more closely aligned towards the amplifier's in-phase axis, Fig. 1b. This effect, known as 'phase squeezing', is inherently suitable for the regeneration of binary phase-encoded signals, as the 'phase squeezed' data bits have a phase of 0 or $\pi$, depending on the input signal.

PSA can be achieved in fibre using interferometric configurations based on nonlinear optical loop mirrors ${ }^{10,14}$, or using FWM processes ${ }^{15}$ implemented in either single or dual-pump configurations. A candidate for such a process is degenerate FWM, as shown in Fig. 1c. Proofof-concept experiments on PSA based regeneration at a data rate of $10 \mathrm{Gbit} / \mathrm{s}$ have recently been demonstrated $^{13}$. The challenge in realizing a practical system lies in the fact that the phase relationship between the pump(s) and the data signal carrier needs to be stabilized at the PSA input. In all FWM based PSA regeneration demonstrations reported to date, all of the phaselocked carriers required were generated prior to imparting data modulation. This is unsuitable for use in a real transmission system where only the data (not the pumps) are transmitted. Consequently, to realize 'black-box' operation, some means to recover the carrier from a carrierless and noisy Phase Shift Keyed (PSK) signal and to phase-lock it to the necessary pumps is required. Furthermore, the previously reported demonstrations of simultaneous amplitude and phase regeneration rely on high-frequency electro-optic modulation to generate multiple phasecoherent beams to be used as signal and pumps ${ }^{15}$, which limits the signal regeneration bandwidths to a few-tens of GHz. 
Our approach ${ }^{16}$ provides amplitude and phase regeneration in a degenerate PSA, however the pumps are generated and phase-locked locally, enabling 'black-box' operation. First, we tap the data signal and send it to the Carrier Recovery/Pump Phase Locking unit, see Fig. 2. In this subsystem, we mix Pump 1 (a free-running laser) with the data and generate an idler wave through a degenerate FWM process in a highly nonlinear fibre (HNLF). The idler phase, $\varphi_{i d l e r}$, fulfills the relation:

$$
\varphi_{\text {idler }}=2 \cdot \varphi_{\text {data }}-\varphi_{\text {Pump } 1},
$$

which brings two important features: it is self phase-locked to the data and the other pump and it does not contain the data pattern, due to the factor of two between the input and output phase of data and the idler phase (the data bits have a phase of 0 [logic ' 0 '] or $\pi$ [logic ' 1 ' $])^{17}$. However, FWM in an optical fibre is an ultra-fast process and thus noise present on the data is transferred to the idler manifesting itself as spectral broadening of the otherwise narrow-linewidth CW idler. The noise can then be eliminated by using a narrowband filtering mechanism, such as laser injection locking. We inject the idler into a semiconductor slave laser and set the injected power to a level at which the signal carrier is tracked at frequencies of up to a few $100 \mathrm{~s}$ of $\mathrm{MHz}^{18}$ without sensitivity to the higher frequency phase noise. Subsequently, we combine the original data stream with the two phase-locked pumps and send them to the PSA operated in saturation for simultaneous phase and amplitude regeneration.

The phase regeneration performance was first characterised using constellation analysis ${ }^{19,} 20$ which provides a very visual representation of how the phase and amplitude noise are affected. Typical data, with the transmitter operating at $10 \mathrm{Gbit} / \mathrm{s}$ (the maximum operating bit-rate of our constellation analyzer), is shown in Fig.3. The regenerator is, however, bit-rate transparent and thus it is believed that any conclusions drawn at $10 \mathrm{Gbit} / \mathrm{s}$ also hold for $40 \mathrm{Gbit} / \mathrm{s}$ or higher daterates. In fact switching between 10 and $40 \mathrm{Gbit} / \mathrm{s}$ did not require any configuration change. The results show that the phase-noise was squeezed by the regenerator to the original (back-to-back) level without degrading the signal amplitude even for extreme differential phase-distortion of \pm 80 deg (peak-to-peak), see Fig. 3 .

The regenerator performance was then assessed at $40 \mathrm{Gbit} / \mathrm{s}$ using eye diagrams and bit error ratio (BER) measurements for various levels of phase-only, amplitude-only and simultaneous amplitude/phase perturbations. The key results are summarized in Fig. 4 showing results for phase-only perturbations for $\pm 30 \mathrm{deg}$ and $\pm 50 \mathrm{deg}$ (Fig. 4a), amplitude-only perturbations for $\pm 25 \%$ and $\pm 50 \%$ (Fig. 4b) and amplitude and phase perturbations when the lower levels of amplitude and phase perturbations were then simultaneously applied (Fig. 4c). Note that the higher levels of the amplitude and phase noise were chosen to be very close to the regenerator limit (over this limit the BER curves are no longer straight lines), as shown in Fig. 4b (green triangles).

The regenerator restores the BER performance to the back-to-back level even for relatively high levels of noise (with noise in the phase, amplitude, or both) that otherwise result in a BER power penalty of more than $10 \mathrm{~dB}$ (at a BER of $10^{-9}$ ), see Fig. 4. In fact, the BER performance is actually improved for modest noise levels as compared to the back-to-back case, which we believe to be due to a slightly non-optimal transmitter configuration. 
The transition to phase-encoded optical communications requires new strategies to cope with phase-related noise. The device presented here directly addresses this issue by removing the phase-noise of phase-encoded signals (performing ultrafast all-optical 1-bit analog-to-digital real-time conversion) while also restoring the signal amplitude. The device was shown to operate on $40 \mathrm{Gbit} / \mathrm{s}$ signals; however it should operate at much higher data rates, limited only by the available pump wavelength separation and dispersion control within the nonlinear fibre used. Our device can also be used as a basic building block for regenerators of multilevel phaseencoded signals - for example, a regenerator for quadruple-phase-shift-keyed signals (QPSK) that incorporates two BPSK regenerator blocks was recently theoretically proposed ${ }^{21}$.

This research has received funding from the European Communities Seventh Framework Programme FP/2007-2013 under grant agreement 224547 (PHASORS) and Science Foundation Ireland under grant agreement 06/IN/I969.

\section{Methods}

The 40 Gbit/s non-return-to-zero (NRZ) differential phase shift keyed (DPSK) data were generated using a narrow linewidth Continuous Wave (CW) fibre laser (a Rock laser from NP Photonics, USA) and a $\mathrm{LiNbO}_{3} \mathrm{Mach}_{\text {ZZehnder }}$ modulator symmetrically driven around zero transmission with $2^{31}-1$ pseudo-random bit sequence (PRBS) data pattern. In order to independently emulate the effects of phase and amplitude noise, the signal was further modulated in a deterministic fashion using two independent modulators: (i) phase modulator driven at a frequency of $\sim 20 \mathrm{GHz}$ and (ii) amplitude modulator driven at frequency of $\sim 1 \mathrm{GHz}$ (both modulators are shown in Fig. 2). The magnitude of the amplitude and phase perturbations was controlled by varying the corresponding modulation depths to emulate different noise levels allowing independent control of the phase and amplitude noise levels, respectively. The distorted signal was then launched into the 'black-box' regenerator.

The local pump laser inside the 'black-box' regenerator (CW Pump 1, Fig. 2) was a single-frequency narrowlinewidth laser (Orbits Lightwave, USA) with a natural linewidth below $1 \mathrm{kHz}$ and an output power of $100 \mathrm{~mW}-\mathrm{its}$ frequency was $200 \mathrm{GHz}$ offset from the data signal carrier frequency.

The slave laser was a discrete mode semiconductor $\operatorname{laser}^{22}$ (Eblana Photonics, Ireland) with an output power of $10 \mathrm{~mW}$ and a natural linewidth below $300 \mathrm{kHz}$. The injected power was close to $-30 \mathrm{dBm}$.

In the 'black-box' regenerator (Fig. 2), a small proportion of the signal was initially tapped off to facilitate the frequency and phase-locking of the two pumps, which occurs in two steps. Firstly, the tapped signal was mixed in a germano-silicate HNLF with the narrow linewidth CW laser (Pump 1) to parametrically generate an idler wave that served as the seed for the second pump. The total power into the HNLF was $\sim 17 \mathrm{dBm}$. The length, dispersion, nonlinear coefficient and attenuation of the HNLF were $500 \mathrm{~m},-0.09 \mathrm{ps} / \mathrm{nm} / \mathrm{km}, 11.5 \mathrm{~W}^{-1} \mathrm{~km}^{-1}$ and $0.8 \mathrm{~dB} / \mathrm{km}$, respectively. Note that due to the phase erasure process, the binary data modulation was not transferred to the idler ${ }^{17}$. Then, the weak idler wave was filtered and injected into a (slave) semiconductor laser (Pump 2) by means of a multiplexer ${ }^{16,23}$. At this stage Pump 1 and Pump 2 were phase-locked to the signal and could serve as pumps in the degenerate PSA (PSA 1). They were then coupled together with the data signal and the three signals were amplified to a total power of $\sim 34 \mathrm{dBm}$, before being launched into an alumino-silicate HNLF for phase regeneration. A strain gradient was applied to the alumino-silicate fibre in order to increase its Stimulated Brillouin Scattering (SBS) threshold to $\sim 29.5 \mathrm{dBm}$ (per CW line) thereby avoiding the need for any active SBS suppression. The HNLF length, dispersion, polarization mode dispersion, nonlinear coefficient and attenuation of the fibre were $177 \mathrm{~m}$, $-0.13 \mathrm{ps} / \mathrm{nm} / \mathrm{km}, 0.11 \mathrm{pskm}^{-0.5}, 7.1 \mathrm{~W}^{-1} \mathrm{~km}^{-1}$ and $15 \mathrm{~dB} / \mathrm{km}$, respectively. The relative powers of the pumps and signals were adjusted for optimal regeneration performance. The total power at the HNLF input was $33 \mathrm{dBm}$. Amplitude regeneration requires operation of the PSA in saturation (requiring relatively high input signal power with respect to the pumps, while phase regeneration requires a high ratio between the maximum gain and deamplification that may be obtained for high pump powers. We found that launching $30 \mathrm{dBm}$ per pump and $20 \mathrm{dBm}$ of signal power resulted in optimum overall performance of the regenerator. These settings correspond to $10 \mathrm{~dB}$ pump to signal power ratio and gave a $20 \mathrm{~dB}$ difference between the maximum gain and de-amplification when measured with no data on the signal. Any slow (sub $\mathrm{kHz}$ ) relative phase drifts between the interacting waves picked 
up due to acoustic and thermal effects present prior to the PSA (e.g., in the first HNLF that generates the seed to the second pump laser) were compensated for by a phase-error tracking scheme (shown as a box in Fig. 2). This subsystem consisted of a second PSA ('tracking PSA', PSA 2) that had signal and pumps relative phase shifted by $\pi / 2$ at its input. Consequently, while maintaining PSA 1 at the maximum phase-sensitive gain for optimal regeneration, PSA 2 was kept at the largest phase-sensitive gain slope, which provides the best tracking performance (by generation of largest error signal). This error signal was sent to an electrical phase-lock loop that controlled a piezoelectric-based fibre stretcher located in the pump path.

The constellation analysis was performed using homodyne coherent detection followed by a real time data analysis (using a $20 \mathrm{GHz}$ real-time oscilloscope from Tektronix providing $50 \mathrm{GSamples} / \mathrm{s}$ ) ${ }^{19,20}$. The local oscillator used in the measurement was obtained by tapping the signal laser before the encoding modulator. From these measurements, bit-to-bit phase-changes were calculated and the corresponding DPSK constellation diagrams generated as shown in Fig. 3. Note that for these measurements, the data rate was adjusted to $10 \mathrm{Gbit} / \mathrm{s}$ and the phase modulator used to degrade the signal was driven at $\sim 5 \mathrm{GHz}$.

\section{References}

1. A. H. Gnauck and P.J. Winzer, "Optical phase-shift-keyed transmission," J. Lightwave Technol. 23 (1), 115-130 (2005).

2. C. Xu, X. Liu, and X. Wei, "Differential phase-shift keying for high spectral efficiency optical transmissions," IEEE J. Sel. Topics Quantum Electron. 10 (2), 281-293 (2004).

3. S.J. Savory, G. Gavioli, R.I. Killey, and P. Bayvel, "Electronic compensation of chromatic dispersion using a digital coherent receiver," Opt. Express 15, 2120-2126 (2007).

4. J. P. Gordon, and L. F. Mollenauer, "Phase noise in photonic communications systems using linear amplifiers," Opt. Lett. 15(23), 1351-1353 (1990).

5. A. Demir, "Nonlinear phase noise in optical-fiber-communication systems," J. Lightwave Technol. 25 (8), 2002-2031 (2007).

6. J. Xu, X. Zhang, Y. Zhang, J. Dong, D. Liu, and D. Huang, "Reconfigurable all-optical logic gates for multi-input differential phase-shift keying signals : design and experiments," J. of Lightwave Technol. 27 (23), 5268-5275 (2009).

7. Z. Tong, A. Bogris, M. Karlsson, and P.A. Andrekson, "Full characterization of the signal and idler noise figure spectra in single-pumped fiber optical parametric amplifiers," Opt. Express 18 (3), 2884-2893 (2010).

8. H.P. Yuen and J.H. Shapiro, "Optical communication with two-photon coherent states," IEEE Trans. Inf. Theory 24 (6), 657-668 (1978).

9. K. Goda, O. Miyakawa, E.E. Mikhailov, S. Saraf, R. Adhikari, K. McKenzie, R. Ward, S. Vass, A. J. Weinstein and N. Mavalvala, "A quantum-enhanced prototype gravitational-wave detector," Nature Physics 4, 472 - 476 (2008).

10. K. Cvecek, K. Sponsel, C. Stephan, G. Onishchukov, R. Ludwig, C. Schubert, B. Schmauss, and G. Leuchs, "Phase-preserving amplitude regeneration for a WDM RZ-DPSK signal using a nonlinear amplifying loop mirror," Opt. Express 16, 1923-1928 (2008).

11. Masayuki Matsumoto and Kenichi Sanuki, "Performance improvement of DPSK signal transmission by a phase-preserving amplitude limiter," Opt. Express 15, 8094-8103 (2007).

12. M. Matsumoto and H. Sakaguchi, "DPSK signal regeneration using a fiber-based amplitude regenerator," Opt. Express 16, 11169-11175 (2008).

13. K. Croussore, I. Kim, Ch. Kim, Y. Han, and G. Li, "Phase-and-amplitude regeneration of differential phase-shift keyed signals using a phase-sensitive amplifier," Opt. Express 14, 2085-2094 (2006). 
14. D. Levandovsky, M. Vasilyev, and P. Kumar, "Amplitude squeezing of light by means of a phase-sensitive fiber parametric amplifier," Opt. Lett. 24 (14), 984-986 (1999).

15. K. Croussore, G.Li, "Phase Regeneration of NRZ-DPSK Signals Based on Symmetric-Pump Phase-Sensitive Amplification", IEEE Photon. Tech. Lett. 19 (11) 864-866 (2007).

16. F.Parmigiani, R. Slavík, J. Kakande, C. Lundström, M. Sjödin, P.A. Andrekson, R. Weerasuriya, S. Sygletos, A. D. Ellis, L. Grüner-Nielsen, D. Jakobsen, S. Herstrøm, R. Phelan, J. O'Gorman, A. Bogris, D. Syvridis, S. Dasgupta, P. Petropoulos, and D. J. Richardson., "All-Optical Phase Regeneration of 40 Gbit/s DPSK Signals in a BlackBox Phase Sensitive Amplifier" in Proc Optical Fiber Communication Conference (OFC/NFOEC 2010), San Diego (USA) Paper PDPC3, (2010).

17. G.-W. Lu, and T. Miyazaki, "Optical phase add/drop for format conversion between DQPSK and DPSK and its application in optical label switching systems," IEEE Photon. Technol. Lett. 21, 322-324, (2009).

18. F. Morgensen, H. Olesen, and g. Jacobsen, "Locking conditions and stability properties for a semiconductor laser with external light injection," IEEE J. of Quantum Electron., QE-21 (7) 784-793 (1985).

19. M. Sjödin, P. Johannisson, M. Sköld, M. Karlsson, and P.A. Andrekson, "Cancellation of SPM in Self-Homodyne Coherent Systems," ECOC 2009, paper We8.4.5.

20. Sköld, J. Yang, H. Sunnerud, M. Karlsson, S. Oda, and P.A. Andrekson "Constellation diagram analysis of DPSK signal regeneration in a saturated parametric amplifier", Opt. Express 16, 5974-5982 (2008).

21. Z. Zhenga, L. Ana, Z. Lia, X. Zhaoa and X. Liu, "All-optical regeneration of DQPSK/QPSK signals based on phase-sensitive amplification", Opt. Communications 281 (10), 2755-2759, 2008.

22. R. Phelan, B. Kelly, J. O'Carroll, C. Herbert, A. Duke, and J. O'Gorman, “ $-40^{\circ} \mathrm{C}<\mathrm{T}<95^{\circ} \mathrm{C}$ mode-hop-free operation of uncooled AlGaInAs-MQW discrete-mode laser diode with emission at $\lambda=1.3 \mu \mathrm{m}$," Electron. Lett. 45 (1), 43-45 (2009).

23. R. Weerasuriya, S. Sygletos, S.K. Ibrahim, R. Phelan, J. O'Carroll, B. Kelly, J. O'Gorman, and A.D. Ellis, "Generation of frequency symmetric signals from a BPSK input for Phase Sensitive Amplification”, OWT6, paper number 1928, OFC 2010. 


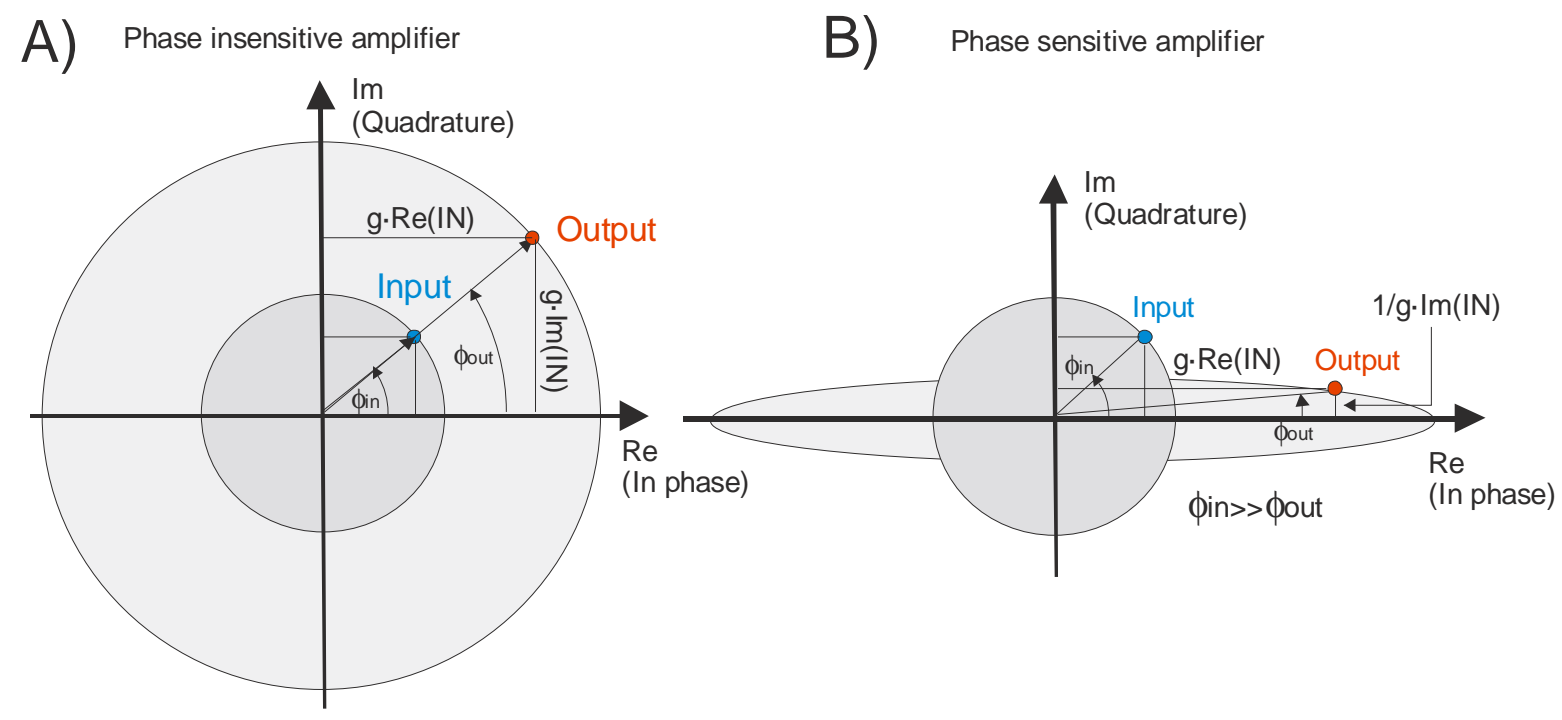

C)

Degenerate four wave mixing phase sensitive amplifier
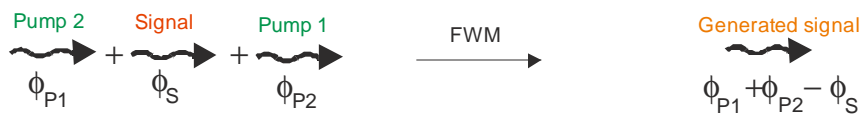

Step 1:

Generation of new signal photons:
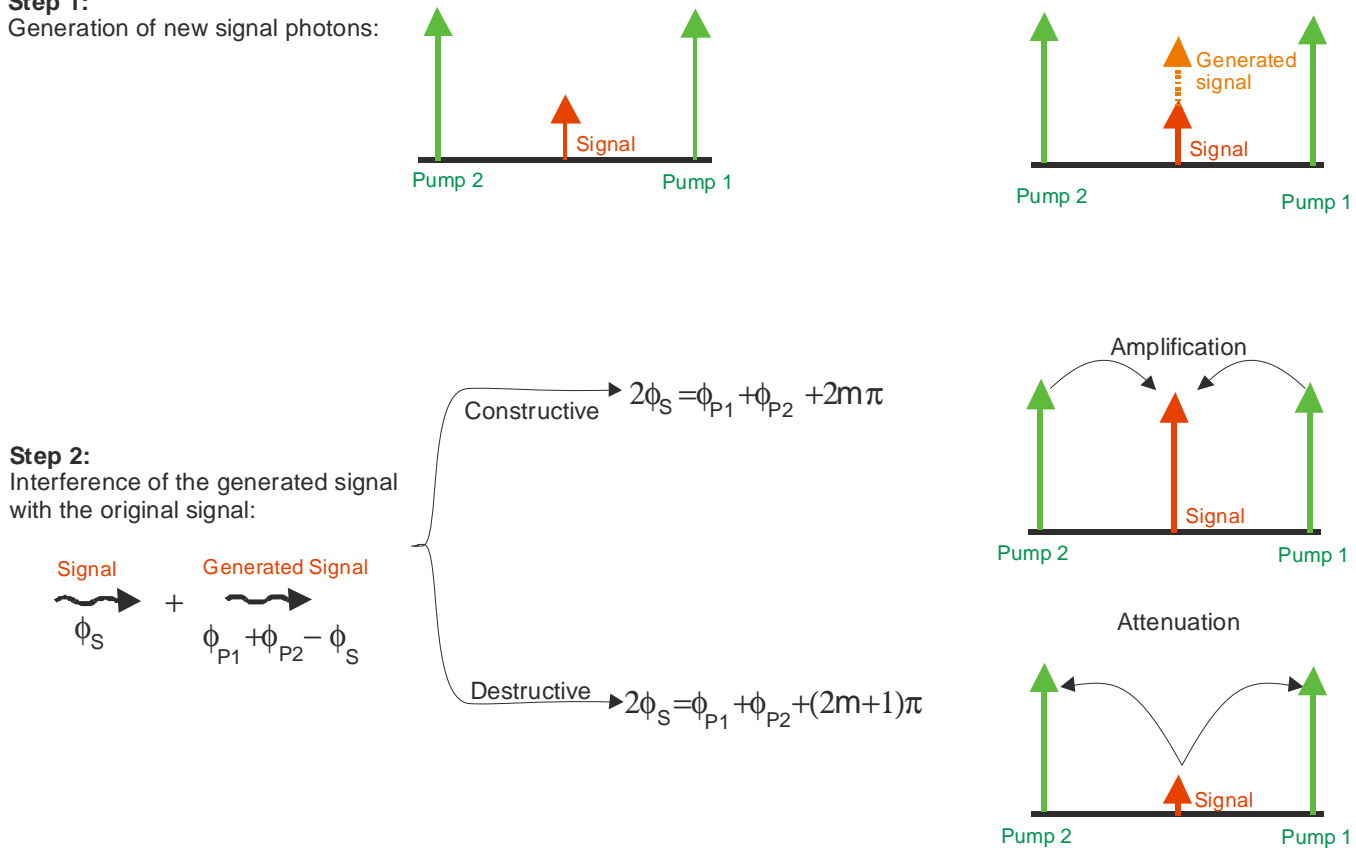

Figure 1: A phase insensitive amplifier maintains the signal phase (A), while the PSA acts to squeeze the phase. One possible implementation of a PSA is through a degenerate four wave mixing process $(\mathrm{C})$ - the PSA only maximally amplifies the signal when the phase of the signal is 0 or $\pi$ relative to the phase of the pumps (in-phase components), and maximally attenuates the signal when this phase is $\pi / 2$ or $3 \pi / 2$. (quadrature components). 


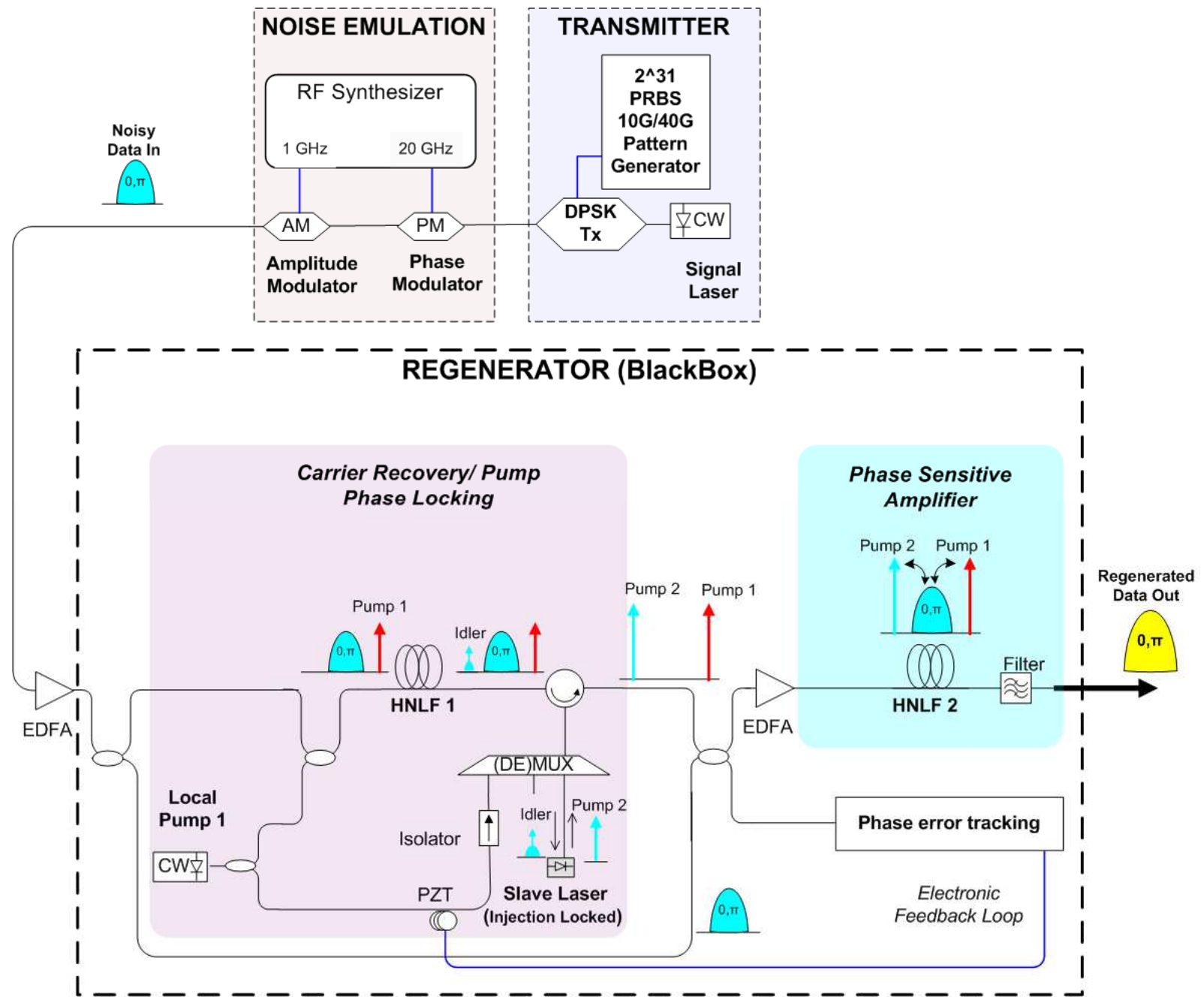

Figure 2: Experimental set-up consisting of the $40 \mathrm{GHz}$ PSK data transmitter, a stage that emulates the amplitude and phase noise (which provides the possibility to add these emulated noise components separately or simultaneously) and the regenerator that has data as its only input (making it 'black-box' style). The 'black-box' consists of two principle units - the first one serves to provide noise-suppressed phase synchronization of the two pumps with the data signal, the second one is a PSA operated in saturation that performs amplitude and phase regeneration. 
Regenerator Input, Output: No added noise
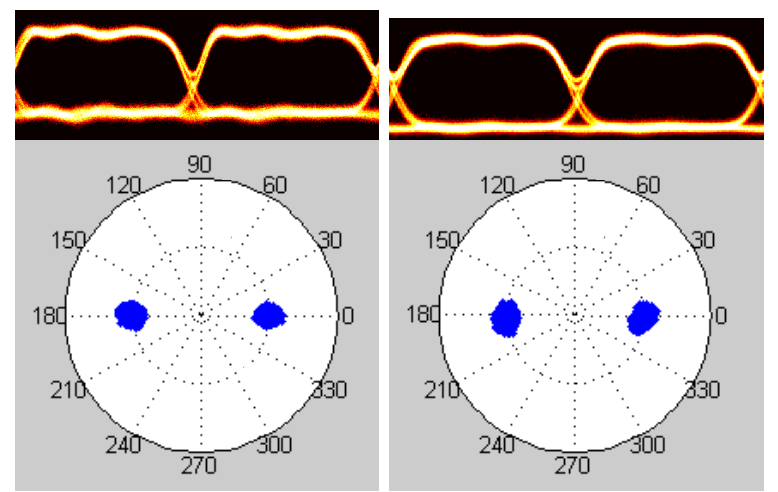

Regenerator Input, Output: High noise level

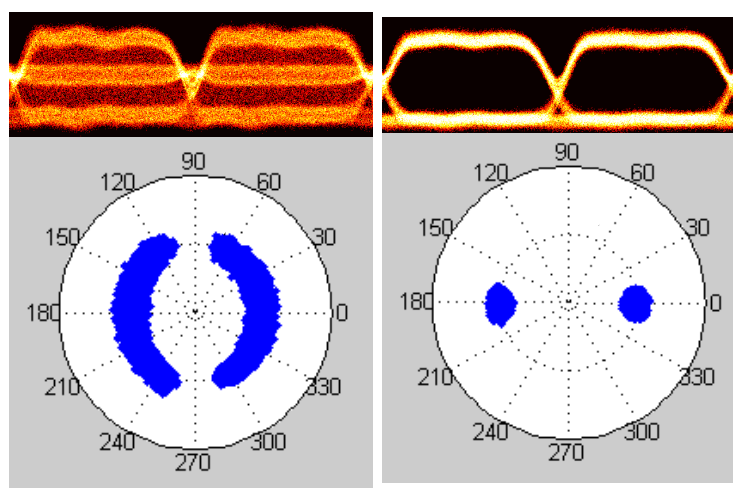

Figure 3: Demodulated eye diagrams after balanced detection and differential constellation diagrams (showing bit-to-bit phase changes) at the input/output of the regenerator measured at $10 \mathrm{Gbit} / \mathrm{s}$. 


\section{A) Phase noise added only}

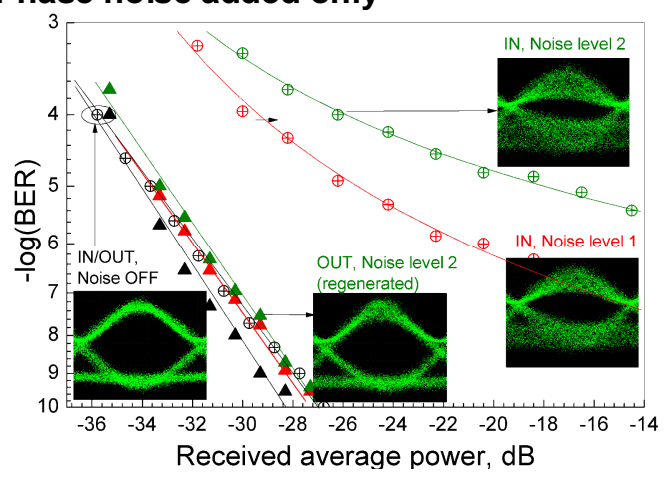

B) Amplitude noise added only

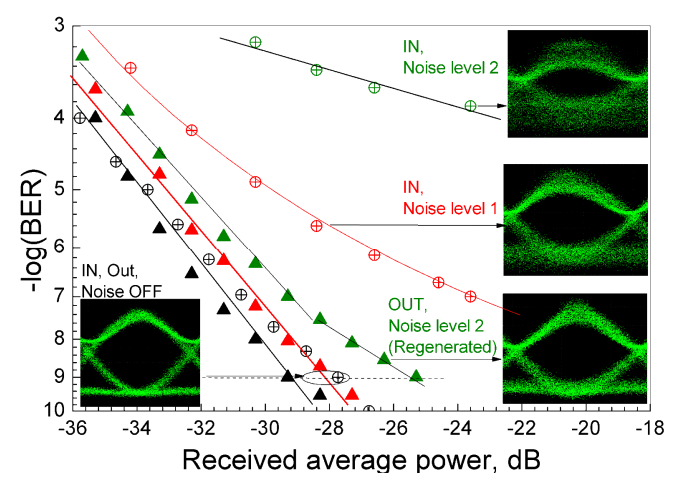

C) Amplitude and phase noise added simultaneously

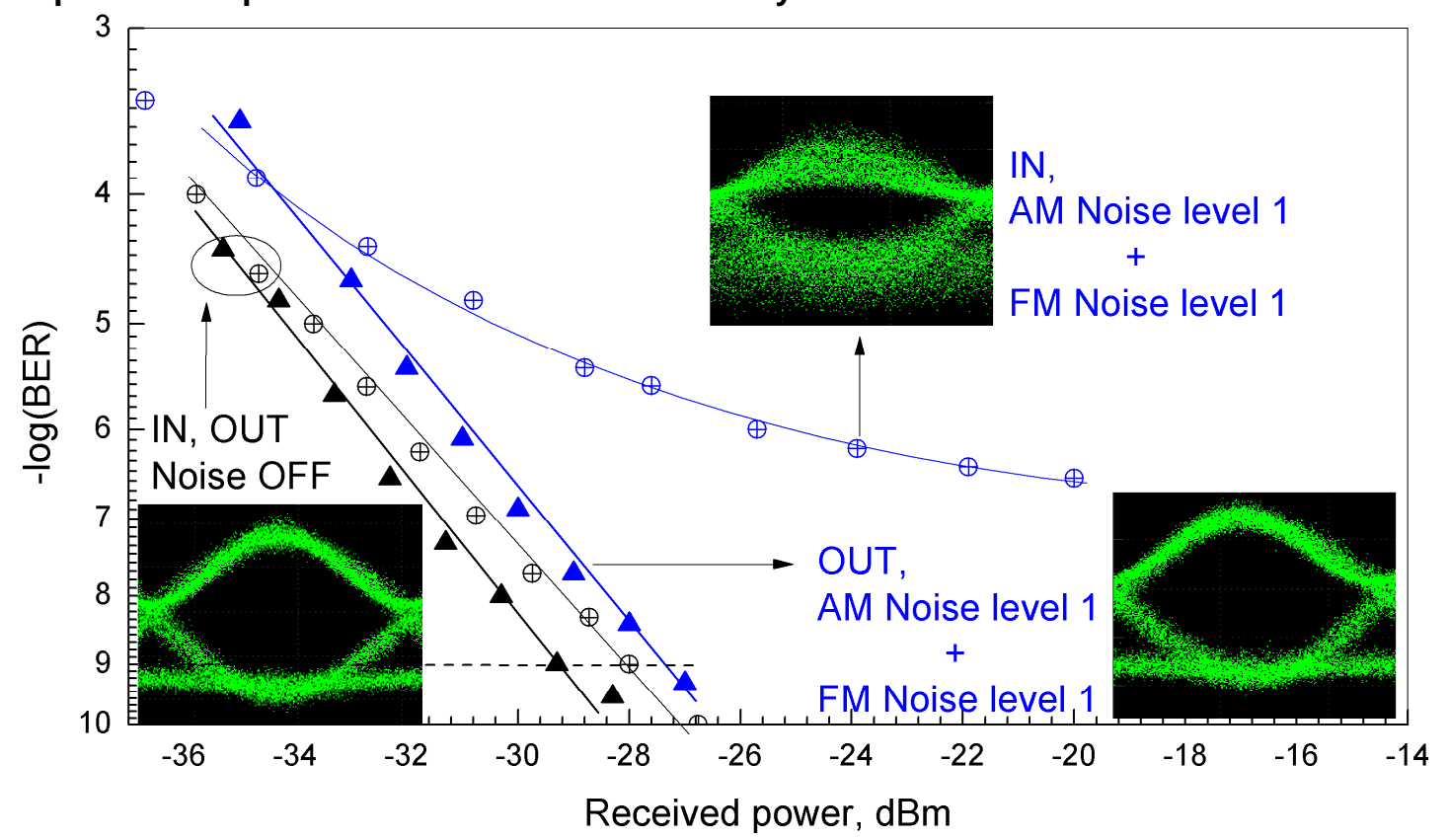

Figure 4 BER curves (measured using single-sided receiver detection after a 1-bit delay line interferometer) and corresponding eye diagrams (measured using a dual-port optical sampling oscilloscope after the 1-bit delay line interferometer) when phase-only (a), amplitude-only (b) and both amplitude and phase (c) noise is added at the input of the system. The performance at the regenerator input and output is shown as circles and triangles, respectively (no noise: black; lower level of amplitude or phase noise: red; higher level of amplitude or phase noise: green; combined (lower level) amplitude and phase noise: blue). 